\title{
Rapid Extraction of Sesquiterpene Lactones from Sage- brush for Use as Taxonomic Markers
}

\author{
RICK G. KELSEY
}

\begin{abstract}
The sesquiterpene lactones of sagebrush occur in glandular trichomes on the leaf surface and can be extracted in 1 to 2 minutes with chloroform. Analysis of this extract by thin-layer chromatography can be used as a taxonomic characteristic.
\end{abstract}

The analysis of sesquiterpene lactones by thin-layer chromatography (Kelsey et al. 1976) can be used as a taxonomic character along with morphology and ecology to help identify or separate species and subspecies of sagebrush (Artemisia, section Tridentatae). Recently it was discovered that these compounds are located in glandular trichomes on the leaf surfaces and can be easily and rapidly isolated with a chloroform wash (Kelsey and Shafizadeh 1980) eliminating the need for the extensive extraction procedures reported previously (Kelsey et al. 1976).

\section{Methods}

Ten to twenty sagebrush leaves from a fresh or air-dried sa mple were placed into a test tube, covered with one milliliter of chloroform, and shaken for 1 to 2 minutes. The leaves were removed, discarded, and the chloroform evaporated from the extract. Evaporation was accelerated by blowing a stream of air over the chloro-

The author is a reseach associate professor, Wood Chemistry Laboratory, Department of Chemistry, University of Montana, Missoula, Montana, 59812

This work was supported by the National Science Foundation PFR 78-826314.

Manuscript received February 17, 1981 form, or placing the test tube into a warm water bath. The concentrated extract was redissolved in a few drops of chloroform before being analyzed by thin-layer chromatography as described earlier (Kelsey et al. 1976).

\section{Discussion}

This new method of isolation has numerous advantages. It requires very little plant material which can be extracted fresh or air-dried. The glassware and quantities of solvent needed is minimal, inexpensive, and easy to obtain. Lastly, the procedure is very rapid so that multiple samples can be prepared in a short period of time. Eighteen to twenty extracts can be chromatographed simultaneously on a single $20 \times 20 \mathrm{~cm}$ chromatography plate in less than two hours, and 20 to 40 specimens can be extracted and chromatographed in one day. This makes the sesquiterpene lactones a much more convenient characteristic to assess for species identification or separation.

\section{Literature Cited}

Kelsey, R.G., M.S. Morris, and F. Shafizadeh. 1976. The use of sesquiterpene lactones as taxonomic markers in the shrubby species of Artemisia (section Tridentatae) in Montana. I. Range Manage. 29:502-505.

Kelsey, R.G., and F. Shafizadeh. 1980. Glandular trichomes and sesquiterpene lactones of Artemisia nova (Asteraceae). Biochem. Syst. Ecol. 8:371-377 